\title{
Identification of Latent Oncogenes with a Network Embedding Method and Random Forest
}

\author{
Ran Zhao, ${ }^{1}$ Bin Hu, ${ }^{2}$ Lei Chen $\mathbb{D}^{1},{ }^{1}$ and Bo Zhou $\mathbb{D D}^{3}$ \\ ${ }^{1}$ College of Information Engineering, Shanghai Maritime University, Shanghai 201306, China \\ ${ }^{2}$ State Key Laboratory of Livestock and Poultry Breeding, Guangdong Public Laboratory of Animal Breeding and Nutrition, \\ Guangdong Provincial Key Laboratory of Animal Breeding and Nutrition, Institute of Animal Science, Guangdong Academy of \\ Agricultural Sciences, Guangzhou 510640, China \\ ${ }^{3}$ Shanghai University of Medicine \& Health Sciences, Shanghai 201318, China
}

Correspondence should be addressed to Lei Chen; chen_lei1@163.com and Bo Zhou; zhoub@sumhs.edu.cn

Received 20 July 2020; Revised 9 September 2020; Accepted 14 September 2020; Published 23 September 2020

Academic Editor: Shijia Zhu

Copyright (C) 2020 Ran Zhao et al. This is an open access article distributed under the Creative Commons Attribution License, which permits unrestricted use, distribution, and reproduction in any medium, provided the original work is properly cited.

\begin{abstract}
Oncogene is a special type of genes, which can promote the tumor initiation. Good study on oncogenes is helpful for understanding the cause of cancers. Experimental techniques in early time are quite popular in detecting oncogenes. However, their defects become more and more evident in recent years, such as high cost and long time. The newly proposed computational methods provide an alternative way to study oncogenes, which can provide useful clues for further investigations on candidate genes. Considering the limitations of some previous computational methods, such as lack of learning procedures and terming genes as individual subjects, a novel computational method was proposed in this study. The method adopted the features derived from multiple protein networks, viewing proteins in a system level. A classic machine learning algorithm, random forest, was applied on these features to capture the essential characteristic of oncogenes, thereby building the prediction model. All genes except validated oncogenes were ranked with a measurement yielded by the prediction model. Top genes were quite different from potential oncogenes discovered by previous methods, and they can be confirmed to become novel oncogenes. It was indicated that the newly identified genes can be essential supplements for previous results.
\end{abstract}

\section{Introduction}

Cancer is the second cause of human deaths in the world, following the cardiovascular disease. Lots of people directly died from cancer per year [1]. Although several efforts have been made in recent years, the mechanism of cancers has not been fully uncovered, which makes difficulties in designing effective treatments. Genetic background and environmental factors are widely accepted to be major causes of cancers [2]. Investigation on the mechanism of cancers with related genes is an essential way to understand the tumor initiation and development.

Oncogene is an important type of cancer-related genes, which can promote the tumor initiation. Thus, it is essential to identify latent oncogenes as much as possible, promoting the understanding of cancers. In early time, experimental techniques performed on typical cell lines or animal models are the main way for detecting oncogenes. However, this way is time-consuming and with high cost. In recent years, with the development of computer science, this procedure can be improved aided by designing computational methods. The computational methods can give a deep insight into a large-scale data and learn hidden associations between cancers and genes, thereby making useful clues and providing latent candidates. Experimenters can do targeted tests to confirm the results. Two pioneer studies have been proposed in this regard recently. The first study proposed a network method for inferring novel oncogenes based on validated oncogenes reported in some online databases [3]. The method applied the shortest path (SP) algorithm on a protein-protein interaction (PPI) network to extract the shortest paths connecting any two proteins of oncogenes. Proteins lying on these paths were picked up and screened by three measurements. 37 possible oncogenes were obtained 
by this method. The second study investigated oncogenes in a quite different way [4]. It tried to uncover the functions, including Gene Ontology (GO) terms and biological pathways, of oncogenes with machine learning algorithms. They first extracted essential GOs and pathways that can indicate the differences of oncogenes and other general genes and made prediction with them. More than 800 genes were predicted to be novel oncogenes. All of the above two studies proposed some putative oncogenes; some of which were extensively discussed. However, the limitations also exit. For the network method proposed in the first study [3], it did not contain a learning procedure, indicated that it cannot capture the essential features of oncogenes, inducing several false positive oncogenes. Although the second method [4] contained a learning procedure, it did not include the protein association information. As proteins with strong associations always share common functions, the protein association information is powerful materials for discovering novel oncogenes.

In view of the limitations of the above two studies, this study proposed a new computational method. The protein networks, derived from protein associations, were constructed, from which informative features were extracted to represent genes. The classic machine learning algorithm, random forest (RF) [5], was adopted to capture essential features of oncogenes and build the model. The latent oncogenes were ranked by a measurement yielded by the proposed method. Top latent oncogenes were quite different from those reported in previous two studies. We also analyzed some top latent oncogenes to confirm their likelihood of being oncogenes.

\section{Materials and Methods}

2.1. Materials. Validated oncogenes were directly downloaded from a previous study [3], which were collected from HUGO Gene Nomenclature Committee (HGNC, https:// www.genenames.org/) [6] and Gene Set Enrichment Analysis Molecular Signatures Database (GSEA MSigDB, https://www .broadinstitute.org/gsea/msigdb/gene_families.jsp) [7, 8]. From HGNC, 251 oncogenes were collected and 330 oncogenes were retrieved from GSEA MSigDB. 543 oncogenes were obtained after combining above two sets of oncogenes. Because we used protein-protein interaction (PPI) networks to identify latent oncogenes, where proteins were represented by Ensembl IDs, proteins encoded by these 543 oncogenes were extracted and they were further mapped onto their Ensembl IDs. After excluding Ensembl IDs that were not in the PPI networks, we finally accessed 481 Ensembl IDs. With these IDs, we designed a computational method to identify new possible IDs. These new IDs suggested latent oncogenes.

\subsection{Protein-Protein Interaction and Network Construction.} In recent years, it is quite popular to adopt networks for investigating various diseases [3, 9-14]. Networks can organize data and information in a system level. It is beneficial to study different problems in a more complete view. Thus, we employed PPI networks to identify novel oncogenes in present study.
In this study, we adopted the PPI data reported in STRING (https://string-db.org/, Version 10.0) [15, 16], a well-known public database collecting known and predicted PPIs. Currently, 9,643,763 proteins from 2,031 organisms comprise huge numbers of PPIs, which were collected from a variety of sources, such as genomic background prediction, high-throughput laboratory experiments, (conservative) coexpression, automated textualization, and prior knowledge in databases. Thus, each interaction contains the physical and functional associations of two proteins and can widely measure the linkage between proteins. Compared with the PPIs reported in other databases, such as DIP (Database of Interaction Proteins) database [17] and BioGRID [18] which only include experimentally validated PPIs, PPIs in STRING contain more information and are more helpful for building models with a complete view. For human, 4,274,001 PPIs are reported in STRING, covering 19,247 human proteins. Each PPI consists of two proteins, encoded by Ensembl IDs. Further, STRING evaluates the strength of each PPI from eight different aspects and assigns eight scores to each PPI, titled by "Neighborhood", "Fusion", "Cooccurrence", "Coexpression", "Experiment", "Database”, “Textmining”, and "Combined_score". The last score combines other seven scores in a naive Bayesian fashion [16]. It was not adopted in the present study because it may produce redundancy with other seven scores. For each of the other scores, one PPI network was constructed in the following manner. We took the "Neighborhood" score as an example. First, 19,247 proteins were picked up as nodes. Second, two nodes were adjacent if and only if the "Neighborhood" score between corresponding proteins was larger than zero. Finally, such "Neighborhood" score was assigned to the corresponding edge as its weight. Accordingly, seven PPI networks were constructed, which were denoted by $N_{N}, N_{F}, N_{\mathrm{CO}}, N_{\mathrm{CE}}, N_{E}, N_{D}$, and $N_{T}$, respectively. The sizes (numbers of edges) of seven networks were quite different. $N_{T}$ had most edges (3816497), followed by $N_{E}$ (1736931 edges), $N_{\mathrm{CE}}$ (768962 edges), $N_{D}$ (212430 edges), $N_{N}$ (76214 edges), $N_{\text {CO }}$ (23739 edges), and $N_{F}$ (2060 edges).

2.3. Feature Engineering. Network is excellent to organize the associations of the proteins, which can view a specific protein in a system level. However, there is a gap between it and the traditional machine learning algorithms because these algorithms always need numerical vectors as input. Fortunately, some network embedding algorithms, such as Mashup [19], Node2vec [20], and Deepwalk [21], were proposed in recent years, which can abstract relationship in the network and output a feature vector for each node in the network. The occurrence of these algorithms connects the network and the traditional machine learning algorithm. Considering the fact that seven networks were involved in this study, Mashup [19] was adopted. It can tackle multiple networks, which is the greatest merit compared with other network embedding algorithms. Its brief descriptions were as follows.

The procedures of Mashup encoding each node consist of two stages. In the first stage, it applies the random walk with the restart (RWR) algorithm [22-26] on each network to construct a raw feature vector for each node in this network. 
In detail, for a network $N_{j}(j \in\{N, F, C O, C E, E, D, T\})$ constructed in Section 2.2, each node $p_{i}$ in this network was picked up as the node seed of the RWR algorithm one by one. When the RWR algorithm stopped, each node in this network was assigned a probability, indicating its associations to the seed node. A raw feature vector of $p_{i}$ was built by collecting all these probabilities, which was denoted by $V_{j}^{i}$. Because some nodes can occur in multiple networks, several feature vectors were generated for these nodes. It is necessary to fuse them into one feature vector in a rigorous way. On the other hand, a dimensionality reduction procedure is also necessary due to the large dimension of the raw feature vectors. All these are the purpose of the second stage of Mashup. Let $X^{i}$ be the final vector of protein $p_{i}$ and $W_{j}^{i}$ be a context feature vector of $p_{i}$ in the network $N_{j}$. It is clear that Mashup tries to determine the optimal components in $X^{i}$ and $W_{j}^{i}$, which retain the essential information in $V_{j}^{i}$ as much as possible. Based on $X^{i}$ and $W_{j}^{i}$, a vector, denoted by $\tilde{V}_{j}^{i}$, can be constructed. Its components were defined as follows:

$$
\tilde{V}_{j k}^{i}=\frac{\exp \left(\left(X^{i}\right)^{T} W_{j}^{k}\right)}{\sum_{k}{ }^{\prime} \exp \left(\left(X^{i}\right)^{T} W_{j}^{k}\right)}, k=1,2, \cdots, n
$$

where $n$ is the total number of different nodes (proteins) in seven networks. The following problem is to find out the optimal components in $X^{i}$ and $W_{j}^{i}$, which can generate $\tilde{V}_{j}^{i}$ approximating $V_{j}^{i}$ as much as possible. An optimization problem is set up to determine the optimal components, which is formulated as below:

$$
\underset{X^{i}, W_{j}^{i}}{\operatorname{minimize}} \frac{1}{n} \sum_{j=1}^{m} \sum_{i=1}^{n} D_{K L}\left(V_{j}^{i} \| \tilde{V}_{j}^{i}\right),
$$

where $m$ stands for the total number of networks and $D_{K L}(\bullet)$ stands for the function of KL-divergence (relative entropy).

The present study used the Mashup program obtained from http://cb.csail.mit.edu/cb/mashup/. The dimension of the output vector is a main parameter of the Mashup. Several dimensions, varying from 100 to 1000 , were tried in this study because we did not know which dimension was best.

2.4. Random Forest. The network embedding algorithm, Mashup, connects the networks and traditional machine learning algorithms. With the feature vectors extracted from seven protein networks via Mashup, a specific machine learning algorithm can deeply study the characteristic of vectors of oncogenes, thereby building a prediction model. This study selected the classic machine learning algorithm, RF [5], due to its wide applications in bioinformatics and medical informatics [27-34].

$\mathrm{RF}$ is an ensemble classification algorithm, which consists of several decision trees. Given a dataset with $n$ samples and $m$ features, RF constructs each decision tree in the following manner. Randomly selected $n$ samples, with replacement, from the original dataset. A decision tree is constructed based on selected samples. When the tree is grown at a node, randomly select $m^{\prime}$ features, where $m^{\prime}$ is much smaller than $m$ , and the optimal splitting way is determined based on these $m^{\prime}$ features. For an input sample, each decision tree first makes its prediction. RF integrates these predictions by majority voting. Although, decision tree is a weak classifier, $\mathrm{RF}$ is deemed to be much strong and competitive compared with other advanced classification algorithms [35].

In this study, a tool "RandomForest" in Weka [36] was directly employed, which implements the above-mentioned RF. Default parameters were used, where the number of decision trees was set to ten.

2.5. The Proposed Method for Inferring Latent Oncogenes. Among the 19,247 proteins occurring in seven protein networks, 481 are encoded by validated oncogenes, whereas the rest 18,766 proteins have not been labelled. It is clear that some proteins may be encoded by latent oncogenes. The proposed method can discover novel latent oncogenes with the feature vectors obtained Section 2.3 and RF described in Section 2.4.

For 18,766 unlabelled proteins, the proposed method evaluated their likelihood of being oncogenes in the following manner. For technique reasons, these 18,766 unlabelled proteins were termed as negative samples, whereas 481 proteins of oncogenes were deemed as positive samples. Evidently, negative samples were much more than positive samples (about 39 times). Thus, we randomly divided the negative samples into 39 parts. Negative samples in each part were combined with positive samples to construct a dataset, thereby yielding 39 datasets. On each dataset, a prediction model was built with RF as the classification algorithm. Accordingly, 39 RF models were produced. For each unlabelled protein $p$, it was fed into 38 RF models, except the RF model containing it. Each model assigned a probability to $p$, suggesting its likelihood to be an oncogene. The mean of all its probabilities was finally calculated to fully measure the likelihood of it being an oncogene. For an easy description, such mean value was called level value.

After all 18,766 unlabelled proteins had been evaluated with the above procedures, we ranked them in a list with the decreasing order of their level values. Evidently, those with high level values were more likely to be oncogenes.

The entire procedures of the method are illustrated in Figure 1.

2.6. Performance Evaluation. To evaluate the utility of the proposed method, a procedure similar to the jackknife test [37-39] was adopted. Each of 481 proteins encoded by oncogenes was singled out one by one as unlabelled proteins. For a specific singled out protein, we want to know whether the rest 480 proteins of oncogenes can identify it. According to the procedures of the abovementioned method, 18,767 unlabelled proteins (one singled out protein and 18,766 actual unlabelled proteins) were also randomly divided into 39 parts. Each part and the positive sample set were combined to generate a dataset, thereby producing 39 datasets. Then, the same procedures of the methods followed to yield the level value of the singled out protein. After all proteins 


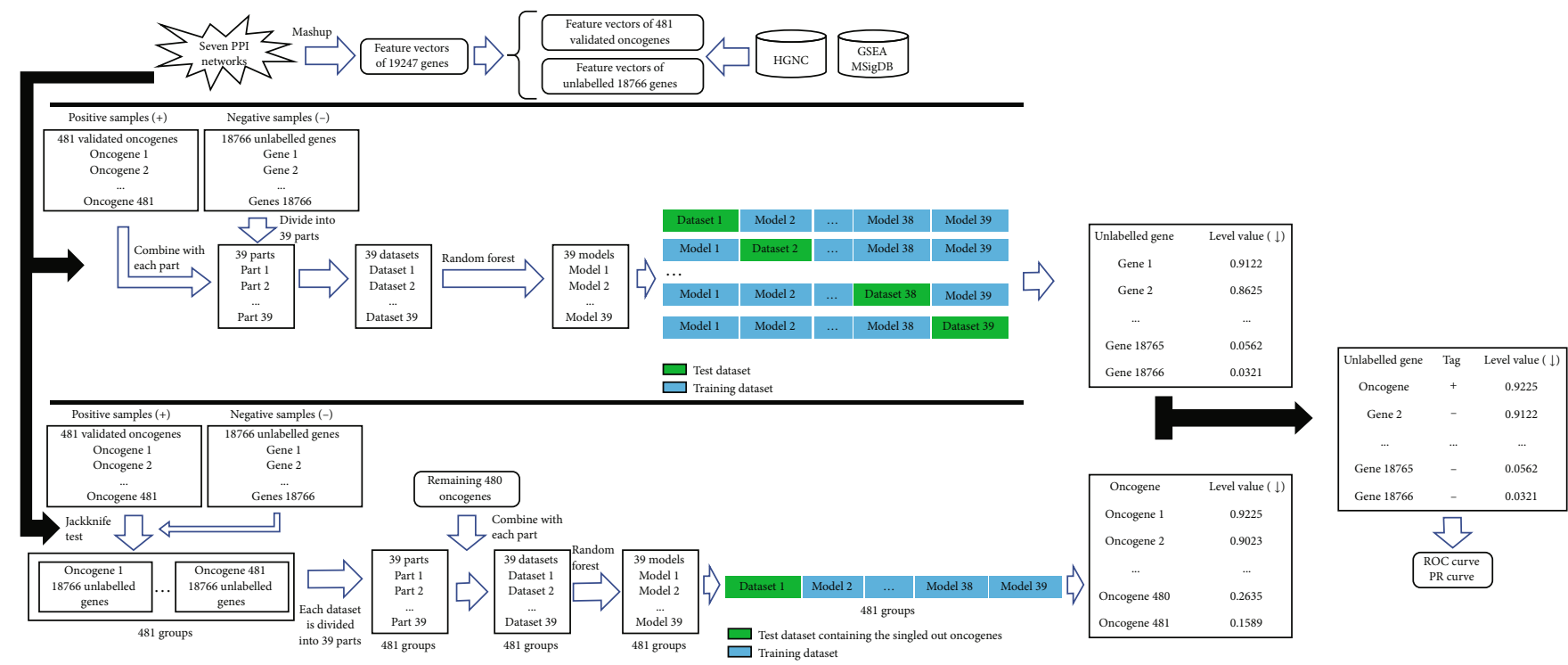

FIGURE 1: Flow chart to show the procedures of the method for inferring latent oncogenes. Seven protein-protein interaction (PPI) networks are constructed using the PPI information reported in STRING, from which feature vectors of oncogenes and unlabelled genes are extracted via Mashup. Unlabelled genes are divided into 39 parts; each of which combines the oncogenes to comprise a dataset. Each dataset induces a random forest (RF) model. A level value is computed for each unlabelled gene, which is the average of the probabilities yielded by $38 \mathrm{RF}$ models. Unlabelled genes are ranked by the decreasing order of their level values. The same procedures are done for each oncogene, thereby ranking oncogenes. Finally, all genes are sorted in a list and ROC and PR curves are plotted to evaluate the performance of the method.

encoded by oncogenes had been tested, they were all assigned a level value. A protein list was created by ranking all 19,247 proteins, including proteins of oncogenes and unlabelled proteins, with the decreasing order of their level values. Some measurements can be calculated to evaluate such list, thereby indicating the utility of the method.

Given a protein list, which sorted proteins with decreasing order of their level values, whether a protein was predicted to be an oncogene (positive sample) was determined after a threshold of level value was set; that is, proteins with level values larger than the threshold were predicted to be oncogenes (positive samples); otherwise, they were predicted to be nononcogenes (negative samples). Accordingly, four values, true positive (TP), false negative (FN), false positive (FP) and true negative (TN), can be counted. Then, the sensitivity (SN) (same as recall), specificity (SP), and precision can be computed by

$$
\begin{gathered}
\mathrm{SN}(\text { recall })=\frac{\mathrm{TP}}{\mathrm{TP}+\mathrm{FN}}, \\
\mathrm{SP}=\frac{\mathrm{TN}}{\mathrm{TN}+\mathrm{FP}}, \\
\text { Precision }=\frac{\mathrm{TP}}{\mathrm{TP}+\mathrm{FP}} .
\end{gathered}
$$

After setting several thresholds, we can obtain a number of SNs, SPs, and precisions. A receiver operating characteristic (ROC) [40] curve and a precision-recall (PR) curve were plotted, where the ROC curve sets $\mathrm{SN}$ as $y$-axis and 1-SP as $x$-axis, whereas PR curve adopts precision as $y$-axis and recall as $x$-axis. Furthermore, the area under each of above two curves can be calculated, which were called AUROC and AUPRC, respectively, in this study. Evidently, the higher the area was, the better the method was.

\section{Results and Discussion}

3.1. Performance of the Method with Different Dimensions. In this study, we used features derived from seven protein networks. Several dimensions were tried to select the best one. For each dimension, the proposed method was evaluated in the way described in Section 2.6. A ROC curve and a PR curve was plotted, as shown in Figure 2. From Figure 2(a), the method with dimension 300 yielded the highest AUROC of 0.8845 , whereas the method with dimension 600 gave the highest AUPRC of 0.4676 from Figure 2(b). In general, the $\mathrm{PR}$ curve is a more accurate measurement than the ROC curve if the dataset is greatly imbalanced. In our study, the negative samples were about 39 times as many as positive samples. Thus, we selected the method with dimension 600 as the proposed method. To further elaborate that this selection is reasonable, we calculated the average of AUROC and AUPRC for each dimension and plotted a scatter diagram to show these averages, as illustrated in Figure 3. Evidently, the dimension 600 gave the highest average of 0.6680 , supporting the above selection. The trend of average on dimension shown in Figure 3 proved the reliability of the results. Before 600 , the average showed an increasing trend, while it generally descended after 600 . It is reasonable because when the dimension was small, several essential information cannot be included, where the dimension was large, lots of noisy was included. All these results supported the method that with dimension 600 was the best choice because it can 

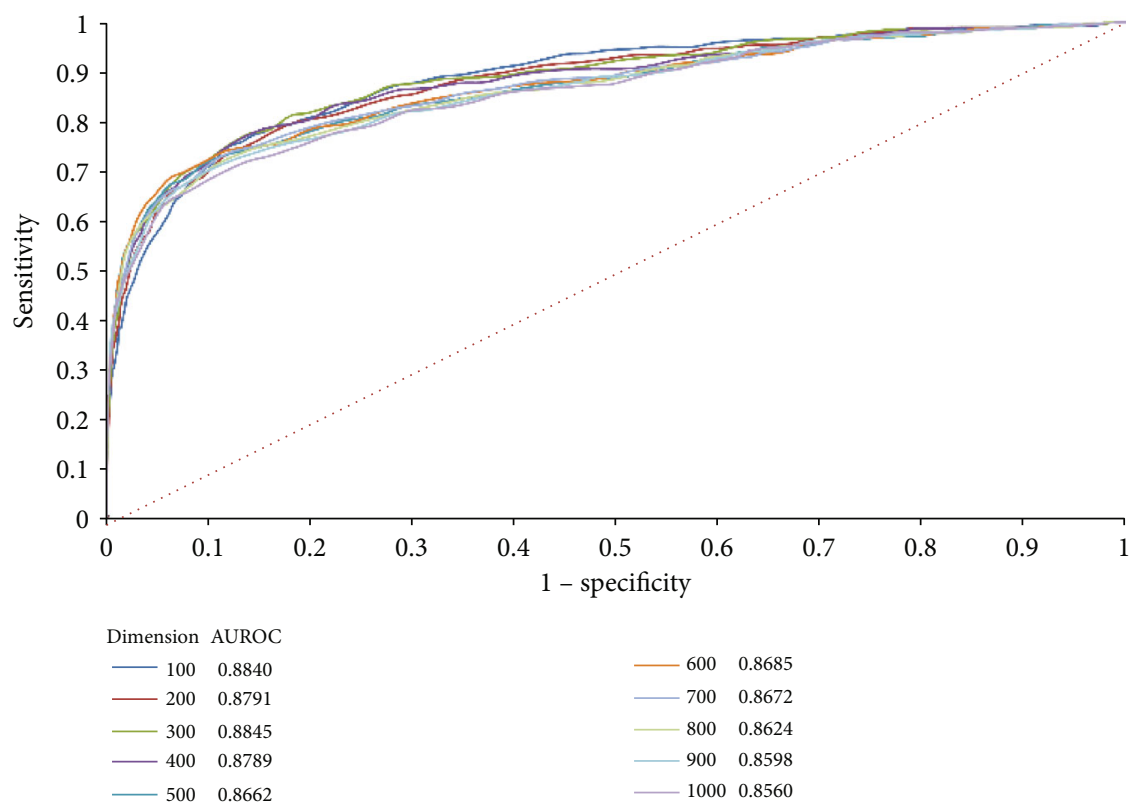

(a)
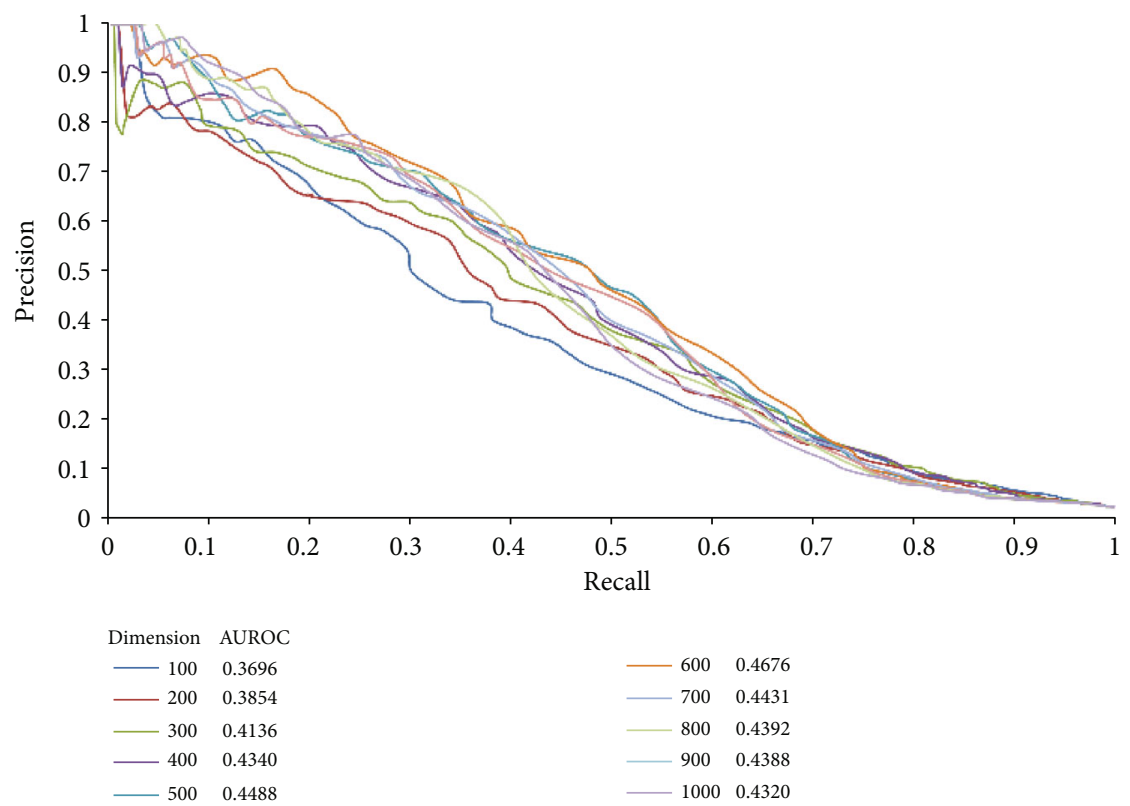

(b)

FIgURE 2: ROC and PR curves to show the performance of the method with different dimensions varying from 100 to 1000 . (a) ROC curves, the dimension 300 yields the highest AUROC of 0.8845; (b) PR curves, the dimension 600 yields the highest AUPRC of 0.4676.

recover actual oncogenes (positive samples) as much as possible. The unlabelled proteins predicted to be positive samples by this method were more reliable.

3.2. Inferred Oncogenes Obtained by the Proposed Method. As mentioned in Section 3.1, we selected the method with dimension 600 as the proposed method. For each unlabelled protein, it was assigned a level value by the method to indicate its likelihood of being oncogenes. The level values of all 18,766 unlabelled proteins are provided in Supplementary Material S1. Figure 4 shows the distribution of all level values.
It can be observed that one unlabelled protein was assigned the level value larger than 0.9 , seven proteins were with level values between 0.8 and 0.9 . These proteins are more likely to be encoded by latent oncogenes. On the other hand, majority proteins (about 96.39\%) received the level values smaller than 0.6.

3.3. Comparison of Previous Studies. Two previous computational methods have been proposed for identifying possible oncogenes. The one method adopted SP algorithm to search novel oncogenes in a PPI network; thus, this method was 


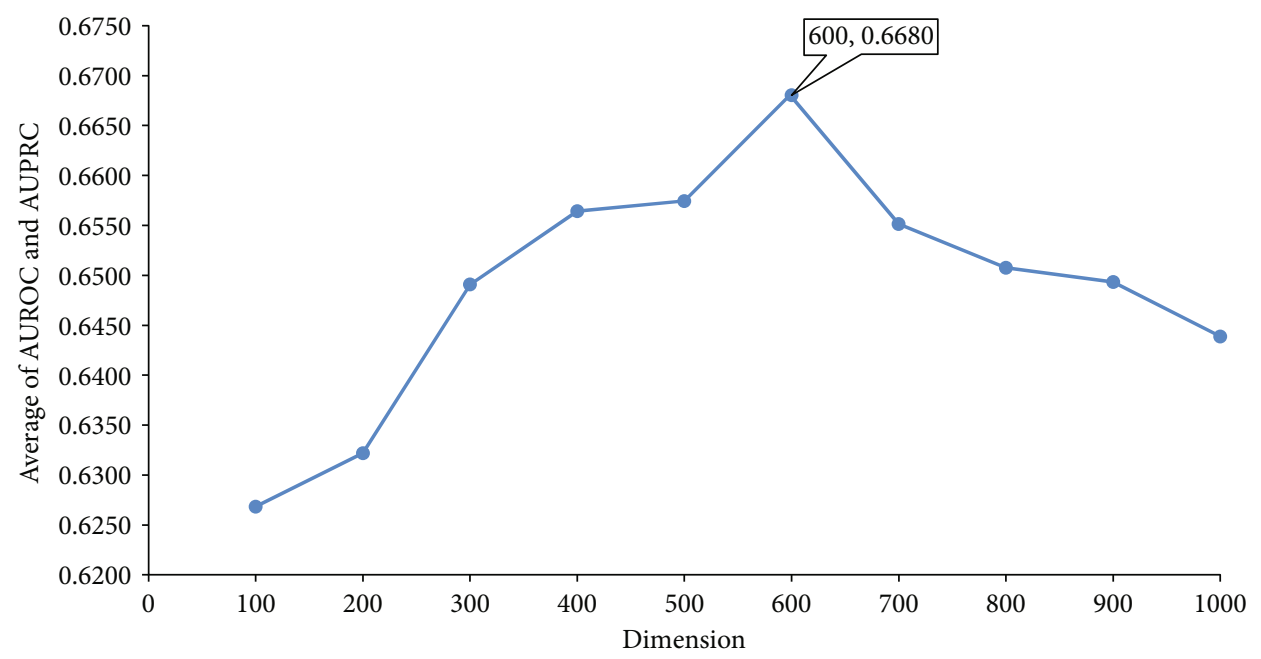

FIGURE 3: Scatter diagram to show the performance of the method with different dimensions varying from 100 to 1000 . The $x$-axis represents dimension and the $y$-axis indicates the average of AUROC and AUPRC. The method with dimension 600 produces best performance.

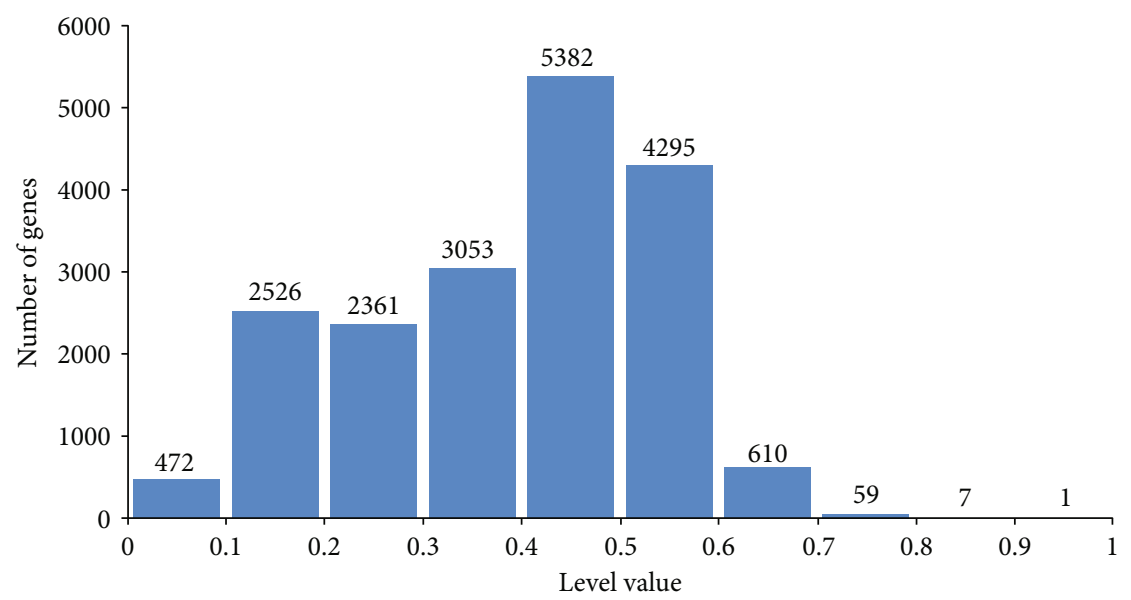

FIGURE 4: Distribution of level values on 18,766 unlabelled proteins. Only 677 unlabelled proteins $(\sim 3.61 \%)$ are assigned the level values higher than 0.6. These proteins are more likely to be encoded by latent oncogenes than others.

called the SP-based method. The other method investigated oncogenes from the point view of their functions; it was termed as function-based method in this study. These previous methods all yielded some latent oncogenes. A comparison was performed in this section.

As our method only ranks the candidates with their level values, we set some thresholds to select inferred genes to make comparisons. The thresholds included $0.8,0.7$, and 0.6 , yielding eight, 67 and 677 inferred oncogenes, respectively. The intersection of these inferred oncogene sets and two oncogene sets yielded by previous methods is illustrated in Figure 5. When the threshold was set to 0.8 , only one gene (HOXA10) was also identified by the SP-based method. 25 inferred oncogenes were shared by either SP- or functionbased methods when the threshold was 0.7 , where two genes (HOXA10, AR) were inferred by all three methods. For the threshold 0.6, this number was 246, where eight genes (HOXA10, AR, ESR1, NOTCH3, PTPN6, MYO5A, KIAA0100, and MAP2K1) were shared by all methods. The exclusive oncogenes yielded by the proposed method occupied $87.5 \%$ when 0.8 was set as the threshold. Such percent was $62.69 \%$ and $63.66 \%$ for the thresholds 0.7 and 0.6 , respectively. These results indicate that majority top inferred oncogenes of our method were not discovered by previous methods, indicating that our method can discover novel latent oncogenes that cannot be identified by previous methods.

3.4. Analysis of Top Inferred Oncogenes. In this study, some latent oncogenes were inferred by the proposed computational method. Each gene was assigned a level value to indicate its likelihood of being oncogenes. Table 1 lists the top fifteen inferred oncogenes. This section selected four of them for detailed analysis.

3.4.1. RAB31. Such gene is the top identification with level value 0.9105. RAB31 (Ras-related protein in brain 31), a member of the RAB family, encodes a protein belonging to 


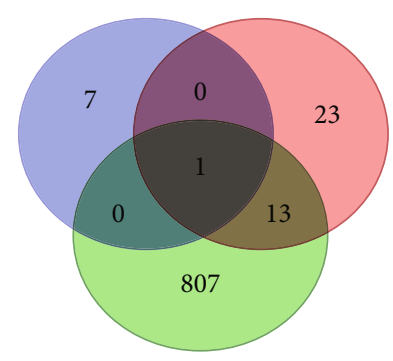

(a)

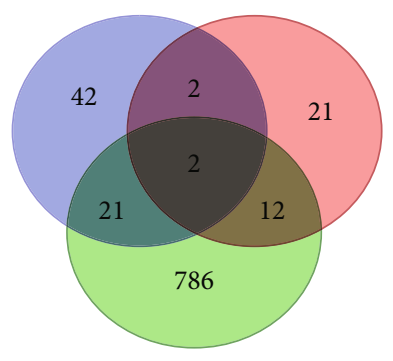

(b)

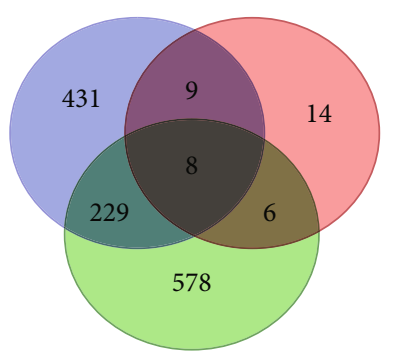

(c)

Figure 5: Venn diagrams to show the intersection of inferred oncogenes yielded by three methods. Blue, red, and green circles represent inferred oncogenes obtained by the proposed method, SP-based method, and function-based method, respectively. (a) Inferred oncogenes with level values larger than 0.8 are considered. (b) Inferred oncogenes with level values larger than 0.7 are considered. (c) Inferred oncogenes with level values larger than 0.6 are considered.

the Ras superfamily of small GTPases. Because it was a significant homology with RAB22 (71\% sequence identity), RAB31 was also named RAB22b. Similar to other members of the RAB family, it functions as molecular switches and plays critical roles in cell adhesion molecules and membrane trafficking of growth factor receptors [41]. Therefore, it is also conceivable that RAB31-mediated dysregulation in endocytosis or recirculation may result in failure to control cell proliferation, adhesion, and migration. As expected, its promotive effect on tumor progression has been reported in several types of cancers [42]. In breast cancer, it was confirmed to be overexpressed in patients with estrogen receptor (ER) positive breast cancer [43]. It is reported that high expression of RAB31 mRNA has a significant correlation with the poor prognosis of lymph node-negative breast cancer patients [44]. Further in vivo and in vitro experiments confirmed that the overexpression of RAB31 promoted cell proliferation of breast cancer cells [45]. Immunohistochemical staining revealed that the expression of RAB31 in liver cancer tissue was significantly higher than that in adjacent liver tissue. Overexpression of RAB31 in liver cancer tissue after hepatectomy is considered to be related to a poor prognosis [46]. In addition, it was found that RAB31 is associated with the survival of glioblastoma [47]. A recent study also confirmed that overexpression of RAB31 in gastric cancer tissues was significantly related to specific clinicopathological features and shorter survival time, strongly suggesting that RAB31 can be a new oncogene for gastric cancer [48].

3.4.2. ACSL5. This gene received the level value of 0.8184 . It encodes a specific transcription factor of the long-chain acyl-CoA synthetase (ACSL) family. In fatty acid metabolism, the first and essential step is the activation of fatty acids. ACSLs, responsible for activation of the most abundant longchain fatty acids (12-20 carbons) in the diet into acyl-CoA thioesters, are generally deregulated in cancer. Such deregulation is also related to poor survival in patients with cancer [49]. The role of ACSL5 in cancer is quite complex. ACSL5 was reported to be downregulated in colorectal carcinomas $[50,51]$, breast cancer $[52,53]$, bladder cancer [53], and pancreas cancer [54]. Furthermore, ACSL5 lower regulation predicted a worse prognosis in breast cancer [52]. However, opposing results were also reported in studies on glioma [55] and gastric cancer [56], where ACSL5 was upregulated.
In addition, fibroblast growth factor receptor 2 (FGFR2) -ACSL5 chimera RNA caused clinical gastric cancer cells to be resistant to the treatment with FGFR inhibitors [57]. Evidences showed that high levels of ACSL5, as a potential downstream target of the transcription factor ONECUT2 (OC2), together with OC2, may cooperatively promote intestinal metaplasia and gastric cancer progression [56]. These contradicting results indicated that the roles of ACSL5 were different among the different cancer types. Lastly, exon 20 skipped variant of ACSL5 protein (splice, Spl) was identified. Results showed that the growth inhibitory effect produced by the Spl protein was opposed to the growth-promoting activity of the nonsplice (NSpl) isoform [58]. Therefore, due to both isoforms, ACSL5 may act either as a tumor suppressor gene or an oncogene.

3.4.3. WNT7B. This gene was assigned a level value of 0.8053 . WNT7B is an extracelluar matrix protein of Wnt family protein [59]. The Wnt (Wingless-INT) was derived from the wingless gene related to visual mutations in Drosophila and the Int1 gene related to mouse breast cancer. Wnt signaling is a well-conserved pathway via canonical ( $\beta$-catenin) and noncanonical (planar cell polarity and calcium) signaling [60]. WNT7B, as an activator of canonical Wnt/ $\beta$-catenin signaling [61], plays a critical role in normal development and tumorigenesis [62]. Because Wnt protein was first isolated from mouse breast cancer, the role of WNT7B on breast cancer has also been increasingly reported. Huguet et al. [63] explored differential expression of human Wnt Genes 2, 3, 4, and $7 \mathrm{~B}$ in human breast cell lines and normal and disease states of human breast tissue. They further found that in $10 \%$ of tumors WNT7B expression was 30 -fold higher than in normal or benign breast tissues. In addition to confirming results consistent with them, Ojalvo et al. [64] and Chen et al. [65] further validated that WNT7B expression makes connections with markers of poor prognosis. Yeo et al. [59] built a Csf1r-icre mouse model using a WNT7B deletion, which also illustrated a critical role of myeloid WNT7B in breast cancer progression, including the levels of angiogenesis, invasion, and metastasis. In addition to breast cancer, abnormal expression of the WNT7b leads to the pathogenesis of many other cancers. Arensman et al. [66] confirmed that WNT7B expression was increased with high activity levels of autocrine Wnt $\beta$-catenin signaling in pancreatic 
TABLE 1: Top fifteen inferred oncogenes.

\begin{tabular}{lcccc}
\hline Rank & Ensembl ID & Gene symbol & Description & Level value \\
\hline 1 & ENSP00000304565 & RAB31 & RAB31, member RAS oncogene family \\
2 & ENSP00000421799 & ENSG00000257184 & - & 0.9105 \\
3 & ENSP00000469872 & RAB4B-EGLN2 & RAB4B-EGLN2 Readthrough (NMD candidate) \\
4 & ENSP00000283921 & HOXA10 & Homeobox A10 & Homeobox D12 \\
5 & ENSP00000385586 & HOXD12 & Acyl-CoA synthetase long chain family member 5 \\
6 & ENSP00000348429 & ACSL5 & RAS-like estrogen regulated growth inhibitor \\
7 & ENSP00000256953 & RERG & Wnt family member 7B & 0.8289 \\
8 & ENSP00000341032 & WNT7B & Ras like without CAAX 2 & 0.8184 \\
9 & ENSP00000321805 & RIT2 & Ras homolog family member C & 0.8184 \\
10 & ENSP00000285735 & RHOC & Fms related receptor tyrosine kinase 1 \\
11 & ENSP00000282397 & FLT1 & DnaJ heat shock protein family (Hsp40) member C27 \\
12 & ENSP00000264711 & DNAJC27 & Acyl-CoA synthetase long chain family member 4 \\
13 & ENSP00000339787 & ACSL4 & Ras like without CAAX 1 & 0.7763 \\
14 & ENSP00000357306 & RIT1 & RHEB like 1 & 0.7763 \\
15 & ENSP00000301068 & RHEBL1 & 0.7763 \\
\hline
\end{tabular}

adenocarcinoma. Zheng et al. [67] found that expression of WNT7B is essential for the growth of prostate cancer cells and this effect is enhanced under androgen-deprived conditions. Their further analyses revealed that WNT7B promotes androgen-independent growth of CRPC cells likely via the activation of protein kinase $\mathrm{C}$ isozymes. Their results further showed that prostate cancer-produced WNT7B maked osteoblast differentiation in vitro and in vivo. As for osteosarcoma (OS) [68], WNT7B expression is dramatically upregulated in OS tissue samples and cells, especially in metastatic OS cell lines. Liu et al. [68] also found that WNT7B silence within OS cells remarkably inhibited the viability and invasion and enhanced the apoptosis of OS cells, suggesting that knocking down WNT7B could inhibit the OS cell growth. Therefore, we presume that WNT7B may function as an oncogene in carcinoma tissue types.

3.4.4. FLT1. This gene was assigned a level value of 0.7763 . Fms-related tyrosine kinase 1 (FLT1, also known as VEGFR-1) is a gene that encodes for a member of the VEGFR family, which presents a critical point in angiogenesis and subsequent cancer progression [69]. The expression of FLT1 is not limited to vascular endothelial cells. It is also found in cells of the hematopoietic lineage (i.e., monocytes and macrophages), dendritic cells, osteoclasts, pericytes, liver cells, placental trophoblasts [70], and smooth muscle cells [71], where it has a regulatory function. Therefore, with respect to carcinogenesis, the role of FLT1 may be more complex [72]. Recent reports also indicate that FLT1 is directly expressed on tumor cells from breast, colon, and skin origin. It is an important oncogenic driver in above cells, boosting survival, cell proliferation, and invasion in an independent manner [73-76]. In head and neck squamous cell carcinoma (HNSSC), FLT1 was selectively overexpressed in tumor tissue. FLT1 was further identified as an important oncogenic driver of HNSCC survival and resistance to radiotherapy with a shRNAmir-based dropout screening setup [72]. Qian et al. [77] found that FLT1 labels a subset of macrophages in human breast cancers which are significantly enriched in metastatic sites. Furthermore, using several genetic models, they elaborated that macrophage FLT1 is important for tumor cell seeding and persistent growth during the distal metastasis. Jiang et al. [78] identified that FLT1 promotes invasion and migration of glioblastoma cells through the modulation of sonic hedgehog ( $\mathrm{SHH}$ ) signaling pathway. A further study has indicated that FLT1 knockdown can prevent the spread of glioblastoma cells in vivo. FLT1 may be a novel oncogene, and therefore, inhibition of FLT1 may serve as a potential target for the development of therapies against metastatic events.

\section{Conclusions}

This study proposed a computational method for the identification of latent oncogenes. From seven protein networks, informative features of proteins were extracted via a powerful network embedding algorithm. Obtained features were learned by random forest, thereby setting up the prediction model. The principle of our method was quite different from previous methods and provided some novel latent oncogenes. Some inferred genes can be confirmed to be novel oncogenes, suggesting that the newly identified oncogenes can be essential supplements for previous studies. It is hoped that the new findings reported in this study can promote the research process of cancers.

\section{Data Availability}

The validated oncogenes were collected from HUGO Gene Nomenclature Committee and Gene Set Enrichment Analysis Molecular Signatures Database. 


\section{Conflicts of Interest}

The authors declare that there is no conflict of interest regarding the publication of this paper.

\section{Authors' Contributions}

Ran Zhao and Bin Hu contributed equally to this work.

\section{Acknowledgments}

This study was supported by the Natural Science Foundation of Shanghai (17ZR1412500), the National Natural Science Foundation of China (61701298), the Key-Area Research and Development Program of Guangdong Province (2018B020203003), Guangzhou science and technology planning project (201707020007), Science and Technology Planning Project of Guangdong Province (2017A010405039).

\section{Supplementary Materials}

Supplementary material S1 Level values of candidate oncogenes. (Supplementary Materials)

\section{References}

[1] S. McGuire, "World Cancer Report 2014. Geneva, Switzerland: World Health Organization, International Agency for Research on Cancer, WHO press, 2015," Advances in Nutrition, vol. 7, no. 2, pp. 418-419, 2016.

[2] F. P. Perera, "Environment and cancer: who are susceptible?," Science, vol. 278, no. 5340, pp. 1068-1073, 1997.

[3] L. Chen, B. Wang, S. P. Wang et al., "OPMSP: a computational method integrating protein interaction and sequence information for the identification of novel putative oncogenes," Protein and Peptide Letters, vol. 23, no. 12, pp. 1081-1094, 2016.

[4] Z. Xing, C. Chu, L. Chen, and X. Kong, "The use of Gene Ontology terms and KEGG pathways for analysis and prediction of oncogenes," Biochimica et Biophysica Acta (BBA) General Subjects, vol. 1860, no. 11, pp. 2725-2734, 2016.

[5] L. Breiman, "Random forests," Machine Learning, vol. 45, no. 1, pp. 5-32, 2001.

[6] K. A. Gray, B. Yates, R. L. Seal, M. W. Wright, and E. A. Bruford, "Genenames.org: The HGNC resources in 2015," Nucleic Acids Research, vol. 43, no. D1, pp. D1079-D1085, 2015.

[7] A. Subramanian, P. Tamayo, V. K. Mootha et al., "Gene set enrichment analysis: a knowledge-based approach for interpreting genome-wide expression profiles," Proceedings of the National Academy of Sciences of the United States of America, vol. 102, no. 43, pp. 15545-15550, 2005.

[8] V. K. Mootha, C. M. Lindgren, K. F. Eriksson et al., "PGC-1 $\alpha$ responsive genes involved in oxidative phosphorylation are coordinately downregulated in human diabetes," Nature Genetics, vol. 34, no. 3, pp. 267-273, 2003.

[9] A. L. Barabasi, N. Gulbahce, and J. Loscalzo, "Network medicine: a network-based approach to human disease," Nature Reviews. Genetics, vol. 12, no. 1, pp. 56-68, 2011.

[10] L. Chen, Z. Xing, T. Huang, Y. Shu, G. H. Huang, and H. P. Li, "Application of the shortest path algorithm for the discovery of breast cancer related genes," Current Bioinformatics, vol. 11, no. 1, pp. 51-58, 2016.
[11] J. Zhang, J. Yang, T. Huang, Y. Shu, and L. Chen, "Identification of novel proliferative diabetic retinopathy related genes on protein-protein interaction network," Neurocomputing, vol. 217, pp. 63-72, 2016.

[12] J. Zhang, Y. Suo, M. Liu, and X. Xu, "Identification of genes related to proliferative diabetic retinopathy through RWR algorithm based on protein-protein interaction network," Biochimica et Biophysica Acta (BBA) - Molecular Basis of Disease, vol. 1864, no. 6, pp. 2369-2375, 2018.

[13] L. Li, Y. S. Wang, L. An, X. Y. Kong, and T. Huang, "A network-based method using a random walk with restart algorithm and screening tests to identify novel genes associated with Menière's disease," PLoS One, vol. 12, no. 8, article e0182592, 2017.

[14] F. Yuan and W. C. Lu, "Prediction of potential drivers connecting different dysfunctional levels in lung adenocarcinoma via a protein-protein interaction network," Biochimica et Biophysica Acta (BBA) - Molecular Basis of Disease, vol. 1864, no. 6, Part B, pp. 2284-2293, 2018.

[15] C. von Mering, M. Huynen, D. Jaeggi, S. Schmidt, P. Bork, and B. Snel, "STRING: a database of predicted functional associations between proteins," Nucleic Acids Research, vol. 31, no. 1, pp. 258-261, 2003.

[16] C. Von Mering, L. J. Jensen, B. Snel et al., "STRING: known and predicted protein-protein associations, integrated and transferred across organisms," Nucleic Acids Research, vol. 33, no. Database issue, pp. D433-D437, 2005.

[17] I. Xenarios, D. W. Rice, L. Salwinski, M. K. Baron, E. M. Marcotte, and D. Eisenberg, "DIP: the database of interacting proteins," Nucleic Acids Research, vol. 28, no. 1, pp. 289-291, 2000.

[18] C. Stark, B. J. Breitkreutz, T. Reguly, L. Boucher, A. Breitkreutz, and M. Tyers, "BioGRID: a general repository for interaction datasets," Nucleic Acids Research, vol. 34, no. 90001, pp. D535-D539, 2006.

[19] H. Cho, B. Berger, and J. Peng, "Compact integration of multinetwork topology for functional analysis of genes," Cell Systems, vol. 3, no. 6, pp. 540-548.e5, 2016.

[20] A. Grover and J. Leskovec, "node2vec: scalable feature learning for networks," in KDD'16: Proceedings of the 22nd ACM SIGKDD International Conference on Knowledge Discovery and Data Mining, pp. 855-864, ACM: San Francisco, CA, USA, 2016.

[21] B. Perozzi, R. Al-Rfou, and S. Skiena, "Deepwalk: online learning of social representations," in KDD '14: Proceedings of the 20th ACM SIGKDD international conference on Knowledge discovery and data mining, New York, NY, USA, 2014.

[22] H. Tong, C. Faloutsos, and J. Pan, "Fast random walk with restart and its applications," in Sixth International Conference on Data Mining (ICDM'06), Hong Kong, China, 2006.

[23] S. Köhler, S. Bauer, D. Horn, and P. N. Robinson, "Walking the interactome for prioritization of candidate disease genes," The American Journal of Human Genetics, vol. 82, no. 4, pp. 949958, 2008.

[24] H. Liang, L. Chen, X. Zhao, and X. Zhang, "Prediction of drug side effects with a refined negative sample selection strategy," Computational and Mathematical Methods in Medicine, vol. 2020, Article ID 1573543, 16 pages, 2020.

[25] L. Chen, T. Liu, and X. Zhao, "Inferring anatomical therapeutic chemical (ATC) class of drugs using shortest path and random walk with restart algorithms," Biochimica et Biophysica 
Acta (BBA) - Molecular Basis of Disease, vol. 1864, no. 6, pp. 2228-2240, 2018.

[26] L. Chen, Y. H. Zhang, Z. Zhang, T. Huang, and Y. D. Cai, "Inferring novel tumor suppressor genes with a proteinprotein interaction network and network diffusion algorithms," Molecular Therapy - Methods \& Clinical Development, vol. 10, pp. 57-67, 2018.

[27] X. Zhao, L. Chen, and J. Lu, "A similarity-based method for prediction of drug side effects with heterogeneous information," Mathematical Biosciences, vol. 306, pp. 136-144, 2018.

[28] X. Zhao, L. Chen, Z. H. Guo, and T. Liu, "Predicting drug side effects with compact integration of heterogeneous networks," Current Bioinformatics, vol. 14, no. 8, pp. 709-720, 2019.

[29] J. R. Li, L. Lu, Y.-. H. Zhang et al., "Identification of synthetic lethality based on a functional network by using machine learning algorithms," Journal of Cellular Biochemistry, vol. 120, no. 1, pp. 405-416, 2019.

[30] S. Wang, D. Wang, J. R. Li, T. Huang, and Y. D. Cai, "Identification and analysis of the cleavage site in a signal peptide using SMOTE, dagging, and feature selection methods," Molecular Omics, vol. 14, no. 1, pp. 64-73, 2018.

[31] E. S. Sankari and D. Manimegalai, "Predicting membrane protein types by incorporating a novel feature set into Chou's general PseAAC," Journal of Theoretical Biology, vol. 455, pp. 319$328,2018$.

[32] L. Wei, P. Xing, J. Tang, and Q. Zou, "PhosPred-RF: a novel sequence-based predictor for phosphorylation sites using sequential information only," IEEE Transactions on Nanobioscience, vol. 16, no. 4, pp. 240-247, 2017.

[33] L. Wei, P. W. Xing, R. Su, G. Shi, Z. S. Ma, and Q. Zou, "CPPred-RF: a sequence-based predictor for identifying cellpenetrating peptides and their uptake efficiency," Journal of Proteome Research, vol. 16, no. 5, pp. 2044-2053, 2017.

[34] Y. B. Marques, A. de Paiva Oliveira, A. T. Ribeiro Vasconcelos, and F. R. Cerqueira, "Mirnacle: machine learning with SMOTE and random forest for improving selectivity in premiRNA ab initio prediction," BMC Bioinformatics, vol. 17, Supplement 18, p. 474, 2016.

[35] M. Fernández-Delgado, E. Cernadas, S. Barro, and D. Amorim, "Do we need hundreds of classifiers to solve real world classication problems?," Journal of Machine Learning Research, vol. 15, no. 1, pp. 3133-3181, 2014.

[36] I. H. Witten and E. Frank, Data mining:practical machine learning tools and techniques, Elsevier, San Francisco, 2nd edition, 2005.

[37] K. C. Chou and C. T. Zhang, "Prediction of protein structural classes," Critical Reviews in Biochemistry and Molecular Biology, vol. 30, no. 4, pp. 275-349, 1995.

[38] J.-P. Zhou, L. Chen, and Z.-H. Guo, "iATC-NRAKEL: an efficient multi-label classifier for recognizing anatomical therapeutic chemical classes of drugs," Bioinformatics, vol. 36, no. 5, pp. 1391-1396, 2020.

[39] L. Chen, W. M. Zeng, Y. D. Cai, K. Y. Feng, and K. C. Chou, "Predicting anatomical therapeutic chemical (ATC) classification of drugs by integrating chemical-chemical interactions and similarities," PLoS One, vol. 7, no. 4, article e35254, 2012.

[40] J. Egan, Signal Detection Theory and ROC Analysis, Academic Press, New York, 1975.

[41] J. Colicelli, "Human RAS superfamily proteins and related GTPases," Science's STKE, vol. 2004, no. 250, article Re13, p. re13, 2004.
[42] C. E. Chua and B. L. Tang, "The role of the small GTPase Rab31 in cancer," Journal of Cellular and Molecular Medicine, vol. 19, no. 1, pp. 1-10, 2015.

[43] M. C. Abba, Y. Hu, H. Sun et al., "Gene expression signature of estrogen receptor $\alpha$ status in breast cancer," BMC Genomics, vol. 6, no. 1, p. 37, 2005.

[44] M. Kotzsch, A. M. Sieuwerts, M. Grosser et al., "Urokinase receptor splice variant uPAR-del4/5-associated gene expression in breast cancer: identification of rab31 as an independent prognostic factor," Breast Cancer Research and Treatment, vol. 111, no. 2, pp. 229-240, 2008.

[45] B. Grismayer, S. Sölch, B. Seubert et al., "Rab31 expression levels modulate tumor-relevant characteristics of breast cancer cells," Molecular Cancer, vol. 11, no. 1, p. 62, 2012.

[46] Y. Sui, X. Zheng, and D. Zhao, "Rab31 promoted hepatocellular carcinoma (HCC) progression via inhibition of cell apoptosis induced by PI3K/AKT/Bcl-2/BAX pathway," Tumour Biology, vol. 36, no. 11, pp. 8661-8670, 2015.

[47] N. V. Serão, K. R. Delfino, B. R. Southey, J. E. Beever, and S. L. Rodriguez-Zas, "Cell cycle and aging, morphogenesis, and response to stimuli genes are individualized biomarkers of glioblastoma progression and survival," BMC Medical Genomics, vol. 4, no. 1, p. 49, 2011.

[48] C. T. Tang, Q. Liang, L. Yang et al., "RAB31 targeted by MiR$30 c-2-3 p$ regulates the GLI1 signaling pathway, Affecting Gastric Cancer Cell Proliferation and Apoptosis," Frontiers in Oncology, vol. 8, p. 554, 2018.

[49] Y. Tang, J. Zhou, S. Hooi, Y.-. M. Jiang, and G.-. D. Lu, "Fatty acid activation in carcinogenesis and cancer development: essential roles of long-chain acyl-CoA synthetases (Review)," Oncology Letters, vol. 16, no. 2, pp. 1390-1396, 2018.

[50] N. Gassler, A. Schneider, J. Kopitz et al., "Impaired expression of acyl-CoA-synthetase 5 in epithelial tumors of the small intestine," Human Pathology, vol. 34, no. 10, pp. 1048-1052, 2003.

[51] F. Hartmann, D. Sparla, E. Tute et al., "Low acyl-CoA synthetase 5 expression in colorectal carcinomas is prognostic for early tumour recurrence," Pathology, Research and Practice, vol. 213, no. 3, pp. 261-266, 2017.

[52] M. C. Yen, J. Y. Kan, C. J. Hsieh, P. L. Kuo, M. F. Hou, and Y. L. Hsu, "Association of long-chain acyl-coenzyme A synthetase 5 expression in human breast cancer by estrogen receptor status and its clinical significance," Oncology Reports, vol. 37, no. 6, pp. 3253-3260, 2017.

[53] N. T. Gaisa, A. Reinartz, U. Schneider et al., "Levels of acylcoenzyme A synthetase 5 in urothelial cells and corresponding neoplasias reflect cellular differentiation," Histology and Histopathology, vol. 28, no. 3, pp. 353-364, 2013.

[54] H. Li, X. Wang, Y. Fang et al., "Integrated expression profiles analysis reveals novel predictive biomarker in pancreatic ductal adenocarcinoma," Oncotarget, vol. 8, no. 32, pp. 5257152583, 2017.

[55] Y. Yamashita, T. Kumabe, Y. Y. Cho et al., "Fatty acid induced glioma cell growth is mediated by the acyl-CoA synthetase 5 gene located on chromosome 10q25.1-q25.2, a region frequently deleted in malignant gliomas," Oncogene, vol. 19, no. 51, pp. 5919-5925, 2000.

[56] E. H. Seo, H. J. Kim, J. H. Kim et al., “ONECUT2 upregulation is associated with $\mathrm{CpG}$ hypomethylation at promoterproximal DNA in gastric cancer and triggers ACSL5," International Journal of Cancer, vol. 146, no. 12, pp. 3354-3368, 2020. 
[57] S. Y. Kim, T. Ahn, H. Bang et al., "Acquired resistance to LY2874455 in FGFR2-amplified gastric cancer through an emergence of novel FGFR2-ACSL5 fusion," Oncotarget, vol. 8, no. 9, pp. 15014-15022, 2017.

[58] I. Pérez-Núñez, M. Karaky, M. Fedetz et al., "Splice-site variant in ACSL5: a marker promoting opposing effect on cell viability and protein expression," European Journal of Human Genetics, vol. 27, no. 12, pp. 1836-1844, 2019.

[59] E. J. Yeo, L. Cassetta, B. Z. Qian et al., "Myeloid WNT7b mediates the angiogenic switch and metastasis in breast cancer," Cancer Research, vol. 74, no. 11, pp. 2962-2973, 2014.

[60] R. Nusse and H. Clevers, "Wnt/ $\beta$-catenin signaling, disease, and emerging therapeutic modalities," Cell, vol. 169, no. 6, pp. 985-999, 2017.

[61] Z. Wang, W. Shu, M. M. Lu, and E. E. Morrisey, "Wnt7b activates canonical signaling in epithelial and vascular smooth muscle cells through interactions with Fzd1, Fzd10, and LRP5," Molecular and Cellular Biology, vol. 25, no. 12, pp. 5022-5030, 2005.

[62] M. Noda, M. Vallon, and C. J. Kuo, “The Wnt7's tale: a story of an orphan who finds her tie to a famous family," Cancer Science, vol. 107, no. 5, pp. 576-582, 2016.

[63] E. L. Huguet, J. McMahon, A. McMahon, R. Bicknell, and A. L. Harris, "Differential expression of human Wnt genes 2, 3, 4, and $7 \mathrm{~B}$ in human breast cell lines and normal and disease states of human breast tissue," Cancer Research, vol. 54, no. 10, pp. 2615-2621, 1994.

[64] L. S. Ojalvo, C. A. Whittaker, J. S. Condeelis, and J. W. Pollard, "Gene expression analysis of macrophages that facilitate tumor invasion supports a role for Wnt-signaling in mediating their activity in primary mammary tumors," Journal of Immunology, vol. 184, no. 2, pp. 702-712, 2010.

[65] J. Chen, T. Y. Liu, H. T. Peng et al., "Up-regulation of Wnt7b rather than Wnt1, Wnt7a, and Wnt9a indicates poor prognosis in breast cancer," International Journal of Clinical and Experimental Pathology, vol. 11, no. 9, pp. 4552-4561, 2018.

[66] M. D. Arensman, A. N. Kovochich, R. M. Kulikauskas et al., "WNT7B mediates autocrine $\mathrm{WNT} / \beta$-catenin signaling and anchorage-independent growth in pancreatic adenocarcinoma," Oncogene, vol. 33, no. 7, pp. 899-908, 2014.

[67] D. Zheng, K. F. Decker, T. Zhou et al., "Role of WNT7Binduced noncanonical pathway in advanced prostate cancer," Molecular Cancer Research, vol. 11, no. 5, pp. 482-493, 2013.

[68] Q. Liu, Z. Wang, X. Zhou et al., "miR-342-5p inhibits osteosarcoma cell growth, migration, invasion, and sensitivity to doxorubicin through targeting Wnt7b," Cell Cycle, vol. 18, no. 23, pp. 3325-3336, 2019.

[69] N. Ferrara, H. P. Gerber, and J. LeCouter, "The biology of VEGF and its receptors," Nature Medicine, vol. 9, no. 6, pp. 669-676, 2003.

[70] M. Shibuya and L. Claessonwelsh, "Signal transduction by VEGF receptors in regulation of angiogenesis and lymphangiogenesis," Experimental Cell Research, vol. 312, no. 5, pp. 549-560, 2006.

[71] Y. Wu, A. T. Hooper, Z. Zhong et al., "The vascular endothelial growth factor receptor (VEGFR-1) supports growth and survival of human breast carcinoma," International Journal of Cancer, vol. 119, no. 7, pp. 1519-1529, 2006.

[72] E. J. Van Limbergen, P. Zabrocki, M. Porcu, E. Hauben, J. Cools, and S. Nuyts, "FLT1 kinase is a mediator of radioresis- tance and survival in head and neck squamous cell carcinoma," Acta Oncologica, vol. 53, no. 5, pp. 637-645, 2014.

[73] F. Fan, J. S. Wey, M. F. McCarty et al., "Expression and function of vascular endothelial growth factor receptor-1 on human colorectal cancer cells," Oncogene, vol. 24, no. 16, pp. 2647-2653, 2005.

[74] T. H. Lee, S. Seng, M. Sekine et al., "Vascular endothelial growth factor mediates intracrine survival in human breast carcinoma cells through internally expressed VEGFR1/FLT1," PLoS Medicine, vol. 4, no. 6, article e186, 2007.

[75] B. M. Lichtenberger, P. K. Tan, H. Niederleithner, N. Ferrara, P. Petzelbauer, and M. Sibilia, "Autocrine VEGF signaling synergizes with EGFR in tumor cells to promote epithelial cancer development," Cell, vol. 140, no. 2, pp. 268-279, 2010.

[76] S. Naik, R. S. Dothager, J. Marasa, C. L. Lewis, and D. PiwnicaWorms, "Vascular endothelial growth factor receptor-1 is synthetic lethal to aberrant $\beta$-catenin activation in colon cancer," Clinical Cancer Research, vol. 15, no. 24, pp. 7529-7537, 2009.

[77] B. Z. Qian, H. Zhang, J. Li et al., "FLT1 signaling in metastasisassociated macrophages activates an inflammatory signature that promotes breast cancer metastasis," The Journal of Experimental Medicine, vol. 212, no. 9, pp. 1433-1448, 2015.

[78] K. Jiang, Y. P. Wang, X. D. Wang et al., "Fms related tyrosine kinase 1 (Flt1) functions as an oncogene and regulates glioblastoma cell metastasis by regulating sonic hedgehog signaling," American Journal of Cancer Research, vol. 7, no. 5, pp. 1164-1176, 2017. 